\title{
OXIDATIVE PHOSPHORYLATION: Kinetic and Thermodynamic Correlation between Electron Flow, Proton Translocation, Oxygen Consumption and ATP Synthesis under Close to In Vivo Concentrations of Oxygen
}

\author{
Baltazar D. Reynafarje ${ }^{1}$ and Jorge Ferreira ${ }^{2}$ \\ 1. Johns Hopkins University School of Medicine, Department of Biological Chemistry, Baltimore, Maryland 21205, USA. \\ 2. Programa de Farmacología Molecular y Clínica, Facultad de Medicina, Universidad de Chile, Independencia 1027, Casilla \\ 70000 Santiago-7, Chile.
}

Correspondence to: Jorge Ferreira, Programa de Farmacología Molecular y Clínica, Facultad de Medicina, Universidad de Chile, Independencia 1027, Casilla 70000 Santiago-7, Chile. E-mail: jferreir@med.uchile.cl, Fax: +56 2735 5580, Tel: +56 29786069.

Received: 2008.04.15; Accepted: 2008.06.05; Published: 2008.06.09

For the fist time the mitochondrial process of oxidative phosphorylation has been studied by determining the extent and initial rates of electron flow, $\mathrm{H}^{+}$translocation, $\mathrm{O}_{2}$ uptake and ATP synthesis under close to in vivo concentrations of oxygen. The following novel results were obtained. 1) The real rates of $\mathrm{O}_{2}$ uptake and ATP synthesis are orders of magnitude higher than those observed under state-3 metabolic conditions. 2) The phosphorylative process of ATP synthesis is neither kinetically nor thermodynamically related to the respiratory process of $\mathrm{H}^{+}$ejection. 3) The ATP/O stoichiometry is not constant but varies depending on all, the redox potential $\left(\Delta E_{h}\right)$, the degree of reduction of the membrane and the relative concentrations of $\mathrm{O}_{2}, \mathrm{ADP}$, and protein. 4) The free energy of electron flow is not only used for the enzymatic binding and release of substrates and products but fundamentally for the actual synthesis of ATP from ADP and Pi. 5) The concentration of ADP that produces half-maximal responses of ATP synthesis $\left(E C_{50}\right)$ is not constant but varies depending on both $\Delta E_{h}$ and $\mathrm{O}_{2}$ concentration. 6) The process of ATP synthesis exhibits strong positive catalytic cooperativity with a Hill coefficient, $n$, of $\sim 3.0$. It is concluded that the most important factor in determining the extent and rates of ATP synthesis is not the level of ADP or the proton gradient but the concentration of $\mathrm{O}_{2}$ and the state of reduction and/or protonation of the membrane.

Key words: Energy transduction, proton gradient, free energy of electron flow and ATP synthesis

\section{Introduction}

The central and most important aspect of the mitochondrial process of energy transduction in aerobic organisms is the mechanism by which the free energy of respiration is transformed into the chemical of ATP. Since the formulation of the chemiosmotic hypothesis [1], it is firmly believed that the processes of electron flow, $\mathrm{H}^{+}$ejection, $\mathrm{O}_{2}$ uptake and ATP synthesis are always kinetically and thermodynamically related. Thus, it is common practice to evaluate the number of molecules of ATP formed per atom of oxygen consumed by simply evaluating the $\mathrm{H}^{+} / \mathrm{O}$ ratio [2], or by solely determining the amount of $\mathrm{O}_{2}$ consumed under state- 3 metabolic conditions [3]. In this context, it is also stated that (a) "electrons do not flow from fuel molecules to $\mathrm{O}_{2}$ unless ATP needs to be synthesized" [4], and (b) the respiratory energy of electron flow is only used to bind $\mathrm{ADP}$ and Pi and to release the spontaneously formed ATP from the catalytic sites of the synthase [5-8]. It is also asserted that the control of electron flux by $\mathrm{O}_{2}$ is minimal and that in a way not specified the phosphorylative process of ATP synthesis controls the flow of electrons through the mitochondrial respiratory chain [9]. We provide here evidence that the process of ATP synthesis does not depend on the vectorial ejection of $\mathrm{H}^{+}$and the magnitude of the proton gradient, but on the net flow of electrons through the entire respiratory chain. Consequently, it is not sufficient to evaluate the energy metabolism of the cell by only determining the $\mathrm{H}^{+} / \mathrm{O}$ ratio in oxygen-pulse experiments [2] or the amount of $\mathrm{O}_{2}$ consumed under state-3 metabolic conditions [3]. It is postulated that the form of energy directly involved in the process of ATP synthesis is not the chemical $(\Delta \mathrm{pH})$ but the electrical $(\Delta \Psi)$ component of the protonmotive force $(\Delta \mathrm{p})$, and that the most important factor in controlling this process is $\mathrm{O}_{2}$ not ADP.

\section{Material and Methods}

\section{Source of Enzymes, Chemicals and Materials}

Mitochondria and sub-mitochondrial particles from rat liver (RLM and SMP) were prepared as 
previously described [10]. Horse-heart-cytochrome c type IV, ATP, ADP, NADH and succinate were products of Sigma Aldrich Co. The "ATP Monitoring Reagent" (a mixture of luciferin and luciferase) was from Bio Orbit. The reagents used to determine the extent of ATP synthesis using the HPLP procedure [11] were all of grade purity. The luminometer was a product of LKB and the fast oxygen electrode, constructed and used as previously described [12, 13], had a $90 \%$ response time of about 10 milliseconds. The air-tight closed reaction chamber of the luminometer was fitted with the $\mathrm{O}_{2}$ electrode and its reference. The output of both the oxygen electrode and luminometer were suitably modified by changing the amperage and/or the voltage and fed into a KIPP and ZONEN multi-channel recorder usually running at a chart speed of $120 \mathrm{~cm} / \mathrm{min}$. The contents of the reaction chamber were stirred with a magnetic bar rotating at about $1000 \mathrm{rpm}$. The standard reaction medium $(1.0 \mathrm{ml}$ of final volume at $25^{\circ} \mathrm{C}$ ) contained $200 \mathrm{mM}$ sucrose, 50 $\mathrm{mM} \mathrm{KCl}, 10 \mathrm{mM} \mathrm{Na}-\mathrm{KPi}$, pH 7.05, $2 \mathrm{mM} \mathrm{MgSO}$, 6.0 $\mu \mathrm{M}$ cytochrome $\mathrm{c}$, and $50 \mu \mathrm{l}$ of a dilution of luciferin/luciferase mixture in $5.0 \mathrm{ml}$ of water. The presence of cytochrome $c$ in the standard medium was necessary to replace the cytochrome $c$ lost during the preparation of SMP. The enzymes were suspended in the reaction mixture and the uptake of $\mathrm{O}_{2}$ and synthesis of ATP determined as described bellow.

\section{Methods to determine the extent and initial rates of ATP synthesis}

The process of ATP synthesis was determined using both a luciferase procedure and a high-pressure column procedure (HPLC). The latter was used to insure that in consecutive reactions the disappearance of the previously formed ATP is due to complete hydrolysis rather than to a reduction of $\mathrm{O}_{2}$ to levels that are much below the $\mathrm{Km}$ of the luciferase for $\mathrm{O}_{2}$ $[14,15]$. True initial rates of ATP synthesis and $\mathrm{O}_{2}$ consumption were simultaneously determined as follows. First, aliquots of either SMP or mitochondria were injected into the closed reaction chamber of the luminometer filled with the standard medium already supplemented with a respiratory substrate. Second, the reaction mixture was incubated for several minutes until every trace of $\mathrm{O}_{2}$ disappeared from the medium. Third, $50 \mu$ of luciferin/luciferase mixture was added and the system further incubated until every trace of both $\mathrm{O}_{2}$ and ATP disappeared from the medium as detected by both the luciferase reaction and the $\mathrm{O}_{2}$ electrode. Fourth, 1 to $10 \mu \mathrm{l}$ of standard medium containing from 2 to 400 nmols of ADP were added into the cell and the system again incubated until the $\mathrm{O}_{2}$ and ATP (contaminating the sample of ADP) added together with the sample of ADP disappeared from the medium. Fifth, the process of oxidative phosphorylation was initiated by injecting from 0.2 to $20 \mu \mathrm{l}$ of air- or $\mathrm{O}_{2}$-saturated medium containing from 0.115 to $30 \mu \mathrm{M} \mathrm{O}_{2}$ (0.23 to 60 nmols $\mathrm{O}$ ) and both ATP synthesis (light emission) and $\mathrm{O}_{2}$ consumption were simultaneously recorded at $120 \mathrm{~cm} / \mathrm{min}$.

The amount of $\mathrm{O}_{2}$ consumed during the net synthesis of ATP was calculated by subtracting the amount of $\mathrm{O}_{2}$ consumed until the net synthesis of ATP ceased from the amount of $\mathrm{O}_{2}$ added at zero time. The amount of ATP formed at the moment the net synthesis of ATP ceases was determined by measuring the distance between the base line and the top of the trace (see Figs. $1 \mathrm{~b}$ and 2). This distance was then compared with standard curves constructed by adding different levels of ATP to air-saturated mediums in the presence and absence of respiratory substrates [16]. The impairing accumulation of oxyluciferin (a product of the luciferase reaction) was prevented by limiting the amount of ATP formed to a maximum of $25 \mu \mathrm{M}$ $[16,17]$.
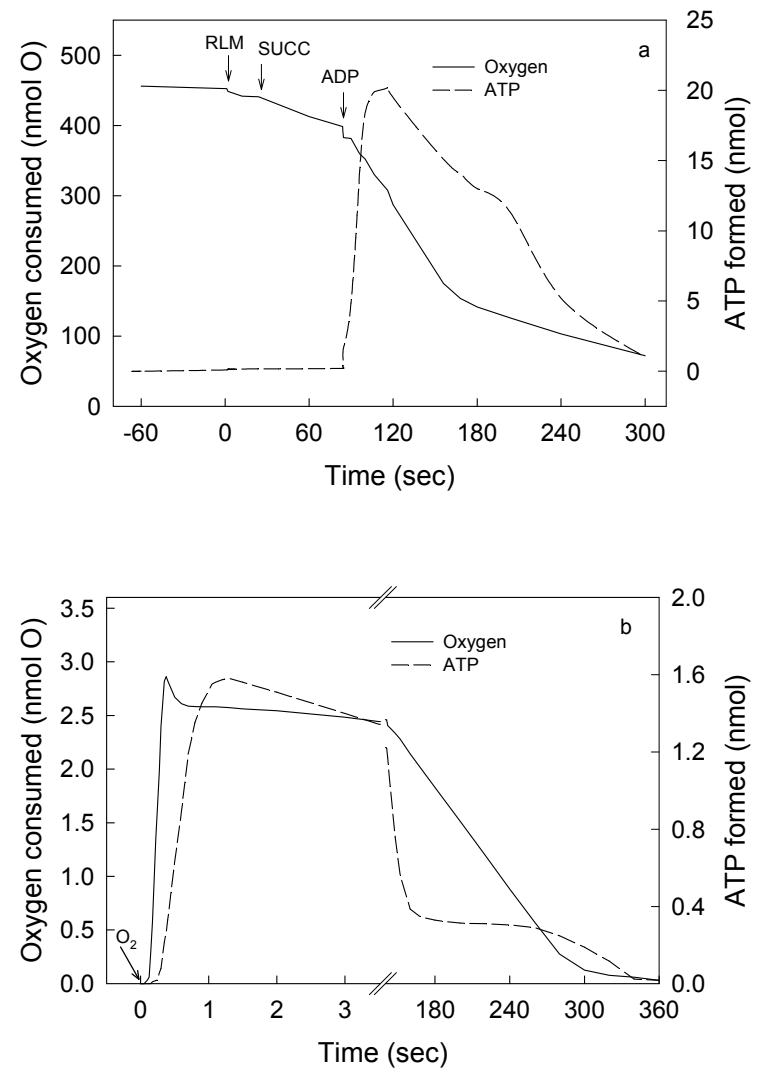

Figure 1. Maximal rates of $\mathrm{O}_{2}$ consumption and ATP synthesis can only occur in reactions catalyzed by a fully reduced mitochondrial membrane. The air-saturated standard reaction medium was that described under Experimental Procedures, with $230 \mu \mathrm{M} \mathrm{O}_{2}, 10 \mathrm{mM}$ succinate and $0.15 \mathrm{mg}$ of RLM protein. In the first portion of this representative experiment (Figs. 1a), 
the reaction was initiated by adding 300 nmols of ADP and the extent and rates of $\mathrm{O}_{2}$ uptake and ATP synthesis simultaneously recorded for $5 \mathrm{~min}$. The reaction was let to continue, unrecorded, for at least $25 \mathrm{~min}$ until both $\mathrm{O}_{2}$ and ATP completely disappeared from the medium (see Experimental procedures). In the second portion of the experiment (Fig. 1b), the reaction was initiated by adding 4.6 nmols of $\mathrm{O}\left(2.3 \mu \mathrm{M} \mathrm{O}_{2}\right)$ to the now fully reduced suspension of mitochondria already in the presence of 300 nmols of ADP. This is a representative experiment of at least four independent determinations.

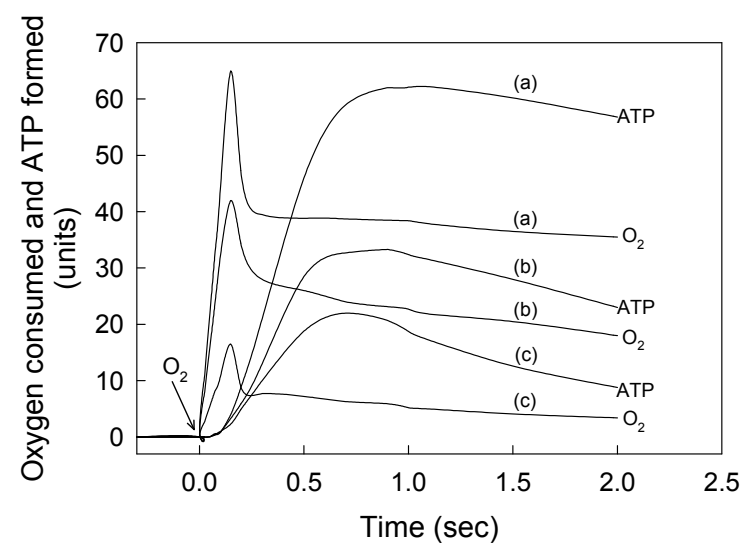

Figure 2. A strict kinetic and stoichiometric correlation between ATP synthesis and $\mathrm{O}_{2}$ uptake only exists during the initial phase of the entire process of oxidative phosphorylation. The standard reaction medium contained $0.02 \mathrm{mg}$ of SMP protein supplemented with $10 \mathrm{mM}$ succinate and $50 \mu \mathrm{M}$ ADP. After the SMP consumed all the $\mathrm{O}_{2}$ and there was no trace of ATP left in the medium (see Fig. 1b) the reactions were initiated by consecutively adding $18.4 \mathrm{nmols}$ of $\mathrm{O}$ in (a), 2.76 in (b) and 0.92 in (c). The time course of both $\mathrm{O}_{2}$ consumption and ATP synthesis were simultaneously recorded during the first 2 seconds of the process of oxidative phosphorylation. Each unit of $\mathrm{O}_{2}$ uptake in the $\mathrm{y}$-axis represents $0.036 \mathrm{nmols}$ of $\mathrm{O}$ for the additions of 0.92 and 2.76 nmols of $\mathrm{O}$, and 0.197 nmols of $\mathrm{O}$ for the addition of 18.4 nmols of O. Each unit of ATP synthesis represents $0.03,0.06$ and $0.2 \mathrm{nmols}$ of ATP for the additions of $0.92,2.76$ and 18.4 nmols of $\mathrm{O}$, respectively. Traces shown are representative of at least three independent determinations of each condition.

The initial rates of ATP synthesis were determined within the first $500 \mathrm{~ms}$ by measuring the steepest portion of the trace. The ATP/O stoichiometry was evaluated during the phase of oxidative phosphorylation in which the processes of ATP synthesis and $\mathrm{O}_{2}$ consumption were kinetically and thermodynamically related (see Figs. $1 \mathrm{~b}$ and 2).

The time-courses of $\mathrm{O}_{2}$ consumption and $\mathrm{H}^{+}$ translocation were simultaneously determined as previously described $[18,19]$. Changes in the redox state of cytochrome aа3 and the related rates of $\mathrm{O}_{2}$ consumption were determined during the first $500 \mathrm{~ms}$ of reactions initiated by adding $\mathrm{O}_{2}$ to fully reduced samples of RLM and purified cytochrome c oxidase
$[13,20]$. The degree of cooperativity between catalytic sites of the synthase was determined at different $\Delta E_{h}$ in the presence of different concentrations of $\mathrm{O}_{2}$ and ADP using the following form of Hill equation:

$$
\log \left(\mathrm{v} / \mathrm{V}_{\max }-\mathrm{v}\right)=n \log [\mathrm{ADP}]-n \log E C_{50}
$$

in which $\mathrm{v}$ represents the fractional velocity of ATP synthesis. The value of $\mathrm{v}$ can range from zero (in the absence of ADP) to 1.0, the $\mathrm{V}_{\max }$ obtained when the fully reduced membrane is in the presence of optimal concentrations of $\mathrm{O}_{2}$, ADP and protein (see below). The Hill coefficient, $n$, or degree of cooperativity between catalytic sites of the synthase, was determined by measuring either the rates of synthesis during the steepest portion of the sigmoidal curve or the amount of ATP formed at the moment the net synthesis of ATP ceases. The concentration of ADP that produces half-maximal responses is evaluated by determining either half-maximal rates $\left(E C_{50}\right)$ or half-maximal extents $\left(K_{0.5}\right)$ of ATP synthesis.

\section{Results and Discussion}

\section{Optimal states of reduction and/or protonation of the mitochondrial membrane are essential for the most efficient processes of oxidative phosphorylation.}

Figure 1 ( $a$ and $b$ ) show the simultaneously and continuously recorded time courses of $\mathrm{O}_{2}$ uptake and ATP synthesis in a reaction catalyzed by RLM under two different states of reduction and/or protonation. In Fig.1a the process of oxidative phosphorylation is initiated by adding $300 \mathrm{nmols}$ ADP to mitochondria respiring in state- 4 in the presence of $\sim 230 \mu \mathrm{M} \mathrm{O}{ }_{2}$ (classic conditions). After $5 \mathrm{~min}$ of reaction, the process of oxidative phosphorylation is let to continue for at least 25 min until $\mathrm{O}_{2}$ and ATP completely disappear from the medium as detected by both the oxygen-electrode and the luciferase reaction (see Methods and Procedures). A non-luminescent procedure was also utilized to insure that the disappearance of ATP was not only due to a level of $\mathrm{O}_{2}$ that is below the $\mathrm{K}_{\mathrm{M}}$ of the luciferase. When both $\mathrm{O}_{2}$ and ATP really disappeared from the medium a pulse of only $2.3 \mu \mathrm{M} \mathrm{O}_{2}$ was injected and the time course of the reaction followed at much higher speeds until a second period of anaerobiosis was attained (Fig. 1b). The data show that the process of oxidative phosphorylation has the following novel characteristics. First, even in the presence of in vivo levels of $\mathrm{O}_{2}(<46 \mu \mathrm{M})$ [21, 22], the rates of ATP synthesis and $\mathrm{O}_{2}$ uptake are orders of magnitude higher in reactions catalyzed by fully reduced RLM than in those catalyzed by mostly oxidized RLM in the presence of $\sim 230 \mu \mathrm{M} \mathrm{O}_{2}$ or state-3 [23]. Thus, although the process of oxidative phosphorylation is oxygen 
dependent throughout the physiological range of oxygen tensions (near zero to $230 \mu \mathrm{M}$ or 150 torr) [24, 25]. Data presented in Fig. $1 \mathrm{~b}$ show that in the presence of only $2.3 \mu \mathrm{M} \mathrm{O}_{2}$ the rate of ATP synthesis $(\sim 1,700$ nmols $\cdot \min ^{-1} \cdot \mathrm{mg}$ protein $\left.{ }^{-1}\right)$ is more than 3 fold higher than in the presence of $\sim 230 \mu \mathrm{M}\left(500 \mathrm{nmols} \cdot \mathrm{min}^{-1}\right.$. $\mathrm{mg}$ protein $^{-1}$ in Fig. 1a). Under state-3 metabolic conditions, the rates of $\mathrm{O}_{2}$ uptake and ATP synthesis are mostly impaired because the reaction is initiated by adding ADP to a mitochondrial membrane that in state- 4 is charged with reactive oxygen species (ROS) and nearly devoid of labile protons [26, 27]. This type of impairment is only "partially reversed by the addition of phosphate and phosphate acceptor" [3]. Distinctly, when the reaction is initiated by adding $\mathrm{O}_{2}$ to either, mitochondria, SMP or intact cells [32] devoid of ROS and fully reduced and/or protonated the steady state rates of $\mathrm{O}_{2}$ uptake and ATP synthesis take place under optimal conditions. In fact, the purpose of the warm-up period that athletes perform just before enter a prolonged physical competition is to get ride of reactive oxygen species at the same time that the mitochondrial membrane attains a state of optimal reduction. Second, only a fraction of the $\mathrm{O}_{2}$ consumed in the entire process of oxidative phosphorylation is actually utilized in kinetic and thermodynamic correlation with the extremely fast phase of ATP synthesis. In fact, Fig. $1 \mathrm{~b}$ shows that from a total of 4.6 nmols of $\mathrm{O}$ consumed in the entire reaction only 2.5 nmols are utilized during the steady-state synthesis of 2.7 nmols of ATP. In Fig. 1a the fraction of $\mathrm{O}_{2}$ consumed in direct correlation with the net synthesis of ATP only occurs during the very initial and elusive portion of state- 3 that passes undetected when the $\mathrm{O}_{2}$ trace is greatly condensed to show the entire time course of the reaction. Third, most of the $\mathrm{O}_{2}$ consumed in the entire process of oxidative phosphorylation occurs during the respiratory period in which the rates of $\mathrm{O}_{2}$ uptake are very low and the previously formed ATP is hydrolyzed in a process that coincides with the re-reduction (not the oxidation) of cytochrome aa (Figs. 1 and 2). In conclusion the result of this experiment demonstrates that a strict kinetic and thermodynamic correlation between $\mathrm{O}_{2}$ consumption and ATP synthesis only occur when the mitochondrial membrane is maximally reduced and/or protonated.

\section{The rates of $\mathrm{O}_{2}$ consumption and ATP synthesis are kinetically and thermodynamically related only during the "active" or fast phase of the respiratory process.}

It is firmly believed that, regardless of the magnitude of the $\Delta E_{h}$ and the concentration of ADP, the extent of ATP synthesis depends directly on the amount of $\mathrm{O}_{2}$ consumed in the entire process of oxidative phosphorylation. The results presented in Fig. 2 and Table 1 show, however, that the net synthesis of ATP only occurs during the "active" respiratory process in which the flow of electrons [28, $29]$ and the reduction of $\mathrm{O}_{2}$ to water $[13,30]$ take place at extremely fast rates. Note that in spite of the very large difference in the amount of $\mathrm{O}_{2}$ totally consumed (from 0.92 to 18.4 nmols $\mathrm{O}$ ) only a fraction of this $\mathrm{O}_{2}$ (from 0.65 to 9.93 nmols $\mathrm{O}$ ) is directly utilized in the net synthesis of ATP (0.65 to 12.27 nmols). Note also that not only the extent but also the initial rates of ATP synthesis $\left(72.9,14.3\right.$ and $4.2 \mu \mathrm{mol} \cdot \mathrm{min}^{-1} \cdot \mathrm{mg}$ protein $\left.{ }^{-1}\right)$ depend on the amount of $\mathrm{O}_{2}$ initially consumed. It is mechanistically significant that even in the presence of extremely low levels of $\mathrm{O}_{2}$, the ATP/O stoichiometry is a direct function of $\mathrm{O}_{2}$ concentration (see Table 1). These findings are supported by observations that both humans and guinea pigs native to high altitude can perform the same type of work or synthesize the same amount of ATP utilizing less $\mathrm{O}_{2}$ than their counterparts from sea level [31, 32]. It must be emphasized that only under absolute resting conditions, i.e. under state- 1 metabolic conditions [3], cells operate under steady-state conditions with a constant and unchanged supply of substrates, $\mathrm{O}_{2}$, and ADP. Under in vivo "active" conditions, however, the extent and rates of ATP synthesis constantly change, depending on the availability of $\mathrm{O}_{2}$ that decreases even along the path of a single capillary. In summary, these results provide evidence that, a) the most important factor in controlling the rate of ATP synthesis is not the level of ADP but rather the level of $\mathrm{O}_{2}$ and, b) the respiratory processes of electron flow and $\mathrm{O}_{2}$ reduction control the phosphorylative process of ATP synthesis and not vice versa as is currently believed [4, 9].

Table 1. Oxygen dependence of the oxidative phosphorylation process of ATP synthesis.

\begin{tabular}{|c|c|c|c|c|}
\hline $\begin{array}{c}\mathrm{O}_{2} \text { totally } \\
\text { Consumed } \\
(\text { nmols O) }\end{array}$ & $\begin{array}{c}\mathrm{O}_{2} \text { initially } \\
\text { Consumed } \\
(\mathrm{nmols} \mathrm{O})\end{array}$ & $\begin{array}{c}\text { ATP } \\
\text { formed } \\
(\mathrm{nmols})\end{array}$ & $\begin{array}{c}\text { Rates of ATP } \\
\text { Synthesis } \\
\left(\mu \mathrm{mols}_{-1} \mathrm{~min}^{-1} \mathrm{mg}\right.\end{array}$ & $\begin{array}{c}\text { ATP/O } \\
\text { Stoichiometry }\end{array}$ \\
\hline $0.92 \pm 0.03$ & $0.65 \pm 0.02$ & $0.65 \pm 0.02$ & $4.2 \pm 0.1$ & $1.00 \pm 0.01$ \\
\hline $2.76 \pm 0.08$ & $1.75 \pm 0.05$ & $1.97 \pm 0.08$ & $14.3 \pm 0.2$ & $1.13 \pm 0.01$ \\
\hline $18.4 \pm 0.5$ & $9.93 \pm 0.13$ & $12.27 \pm 0.18$ & $72.9 \pm 1.4$ & $1.24 \pm 0.02$ \\
\hline
\end{tabular}

Note: Experimental conditions were as those described for Fig. 2. The amounts of ATP formed were determined at the moment in which both the net synthesis of ATP and the fast phase of $\mathrm{O}_{2}$ consumption ceased. The initial rates of ATP synthesis were determined within the first $300 \mathrm{~ms}$ of reaction. Values are arithmetical means $\pm \mathrm{SD}_{\mathrm{n}-1}$ of at least three independents determinations 
III. The phosphorylative process of ATP synthesis is neither kinetically nor thermodynamically related to the respiratory process of $\mathrm{H}^{+}$ejection.

In accordance with the chemiosmotic hypothesis [1] it is firmly believed that the processes of electron flow, $\mathrm{H}^{+}$ejection, $\mathrm{O}_{2}$ consumption and ATP synthesis are all kinetically and thermodynamically related. Consequently, the extent of ATP synthesis is usually determined by measuring either the $\mathrm{H}^{+} / \mathrm{O}$ ratio [2] or the amount of $\mathrm{O}_{2}$ consumed under state- 3 metabolic conditions [3]. Until now, however, no attention has been paid to the fact that all, the flow of electrons, the consumption of $\mathrm{O}_{2}$ and the over all process of oxidative phosphorylation are polyphasic in nature $[13,28,30]$. In fact, data compiled in Fig. 3 show that the vectorial process of $\mathrm{H}^{+}$ejection $[18,19]$, is neither kinetically nor thermodynamically related to the flow of electrons, the net oxidation of cytochrome $\mathrm{aa}_{3}$, the consumption of $\mathrm{O}_{2}$ and the net synthesis of ATP. Note that the net ejection of $\mathrm{H}^{+}$, as determined under optimal oxygen-pulse conditions [18, 19, 33], only begins to occur during the respiratory process in which the rates of $\mathrm{O}_{2}$ consumption are very slow and the cytochrome aa 3 undergoes net reduction. The lack of stoichiometric correlation between the vectorial ejection of $\mathrm{H}^{+}$and the processes of $\mathrm{H}^{+}$uptake, $\mathrm{O}_{2}$ consumption and ATP synthesis has also been demonstrated in reactions catalyzed by both paracoccus denitrificans and purified cytochrome $a_{3}[30,34]$. These results show that the most important factor in controlling the synthesis of ATP is not ADP, but $\mathrm{O}_{2}$ and that the proton gradient generated by the respiratory process of $\mathrm{H}^{+}$ejection is not directly related to the actual process of ATP synthesis.

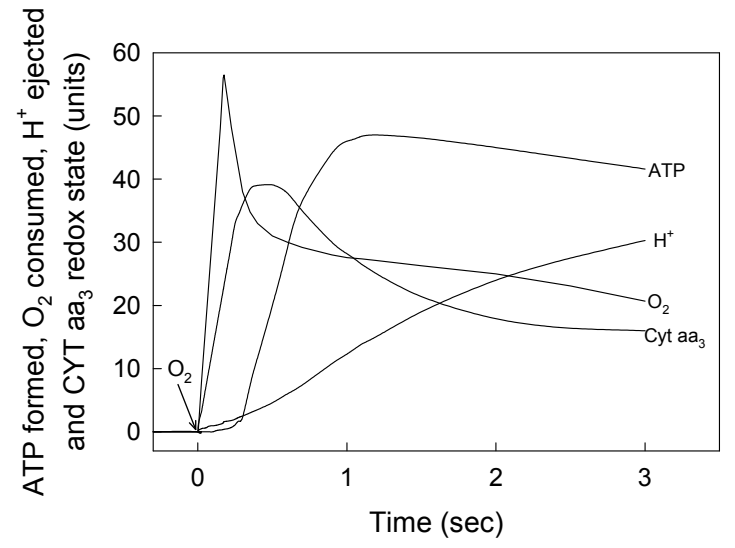

Figure 3. The vectorial ejection of $\mathrm{H}^{+}$is neither kinetically not stoichiometrically related to the processes of cytochrome $\mathrm{aa}_{3}$ oxidation, $\mathrm{O}_{2}$ uptake and ATP synthesis. The basic medium was identical to that described under Experimental Procedures. All the reactions were performed in oxygen-pulse experiments by adding $\mathrm{O}_{2}$ to fully reduced samples of RLM. The uptake of $\mathrm{O}_{2}$ and the changes in the redox state of cytochrome $\mathrm{aa}_{3}$ were simultaneously initiated by adding 9.2 nmols of $\mathrm{O}$ to a fully reduced suspension of $3.5 \mathrm{mg}$ of mitochondrial protein. Every unit in the $\mathrm{y}$-axis represents 0.24 nmols of $\mathrm{O}$ and a $\Delta \mathrm{A}$ of $1.2 \mathrm{x}$ $10^{-4}$ at $605-630 \mathrm{~nm}$. The ejection of vectorial $\mathrm{H}^{+}$was determined by adding $55 \mathrm{nmols}$ of $\mathrm{O}$ to a fully reduced sample of $4 \mathrm{mg}$ of mitochondrial protein. Every unit in the y-axis represents 3.37 nmols of $\mathrm{H}^{+}[18,19]$. The synthesis of ATP was initiated by adding 4.6 nmols of $\mathrm{O}$, like in Fig. 1b. Every unit in the y-axis represents $0.036 \mathrm{nmols}$ of ATP. Traces correspond to representative experiments of at least three independent determinations.

\section{The ATP/O stoichiometry is a function of all, the $\Delta E_{h}$, the redox state of the membrane and the levels of $\mathrm{O}_{2}$, ADP and protein.}

The consensus is that the ATP/O stoichiometry is a constant the value of which only depends on the magnitude of the $\Delta E_{h}$. The results presented in Fig. 4 show, however, that under close to in vivo concentrations of $\mathrm{O}_{2}$, i.e. below $36 \mu \mathrm{M} \mathrm{O}_{2}$ or 23 torr [21, 22], the number of molecules of ATP formed per atom of $\mathrm{O}_{2}$ consumed varies depending on all, the $\Delta E_{h}$ and the relative concentrations of $\mathrm{ADP}, \mathrm{O}_{2}$ and protein. In fact, Fig. 4a shows that in the presence of NADH (a high $\left.\Delta E_{h}\right)$ and $100 \mu \mathrm{M} \mathrm{ADP}$, the $\mathrm{ATP} / \mathrm{O}$ ratio increases from $\sim 1.0$ to a maximum of 3.39 when the concentration of $\mathrm{O}_{2}$ increases from 0.23 to $15.0 \mu \mathrm{M}$. At the same $\Delta E_{h}$ but in the presence of $25 \mu \mathrm{M}$ ADP, the $\mathrm{ATP} / \mathrm{O}$ ratio increases from 0.1 to only 1.87 . In the same range of $\mathrm{O}_{2}$ concentrations but in the presence of cytochrome c (low $\Delta E_{h}$ ) and $100 \mu \mathrm{M}$ ADP the ATP/O ratio remains close to the maximum of 1.33 . In the presence of $25 \mu \mathrm{M} A D P$, however, the $\mathrm{ATP} / \mathrm{O}$ ratio increases from near zero to only 0.126 . Figure $4 \mathrm{~b}$ shows that not only the total amount of ATP formed (Fig. 4a) but also the initial rates of ATP synthesis vary intricately depending on all, $\Delta E_{h}, \mathrm{O}_{2}$ and ADP. Thus, in the presence of NADH and $100 \mu \mathrm{M}$ ADP the initial rates of ATP synthesis increase from near zero to 214 $\mu \mathrm{mol} \cdot \mathrm{min}^{-1} \cdot \mathrm{mg}$ prtoein ${ }^{-1}$ when the level of $\mathrm{O}_{2}$ increases from 0.92 to 23 nmols $\mathrm{O}(0.46$ to $11.5 \mu \mathrm{M})$. In the presence of $\mathrm{NADH}$ and only $25 \mu \mathrm{M}$ ADP the rates increase from less than 1.0 to only $60.7 \mu \mathrm{mol} \cdot \mathrm{min}^{-1}$. $\mathrm{mg}$ prtoein ${ }^{-1}$. In the same range of $\mathrm{O}_{2}$ concentrations $(0.46$ to $11.5 \mu \mathrm{M})$, but in the presence of cytochrome $\mathrm{c}$ and $100 \mu \mathrm{M}$ ADP the rates of ATP synthesis increase from less than 3.78 to a near maximum of $61.4 \mu \mathrm{mol}$. $\mathrm{min}^{-1} \cdot \mathrm{mg}$ protein ${ }^{-1}$. Under the same conditions but in the presence of only $25 \mu \mathrm{M}$ ADP the rates increase from near zero to only $12.3 \mu \mathrm{mol} \cdot \mathrm{min}^{-1} \cdot \mathrm{mg}$ protein ${ }^{-1}$. Figure $4 \mathrm{c}$ show that the net synthesis of ATP depends not only on the $\Delta E_{h}$ and the initial concentrations of $\mathrm{O}_{2}$ and ADP but on the concentration of protein as well. Unexpectedly however, the data show that the extent 
of ATP synthesis decreases as the concentration of protein increases. This odd effect of protein is explained considering that the effective number of collisions between $\mathrm{O}_{2}$ and cytochrome aa depends directly on the molar ratio between $\mathrm{O}_{2}$ and protein. Thus, when the concentration of protein is increased maintaining constant the concentration of $\mathrm{O}_{2}$, the energy directly involved in the synthesis of ATP is substantially reduced. Indeed, the real ATP/O stoichiometry is not a constant but varies exquisitely depending on a large array of factors, amongst which, the most important is the level of oxygen. The reproducibility of the data was confirmed in more than 5 independent experiments by determining the arithmetical means $\pm \mathrm{SD}_{\mathrm{n}-1}$ using a fixed parameter and changing the rest. The " $P$ " value was $<0.05$ for most levels of $\mathrm{O}_{2}$, ADP and protein.

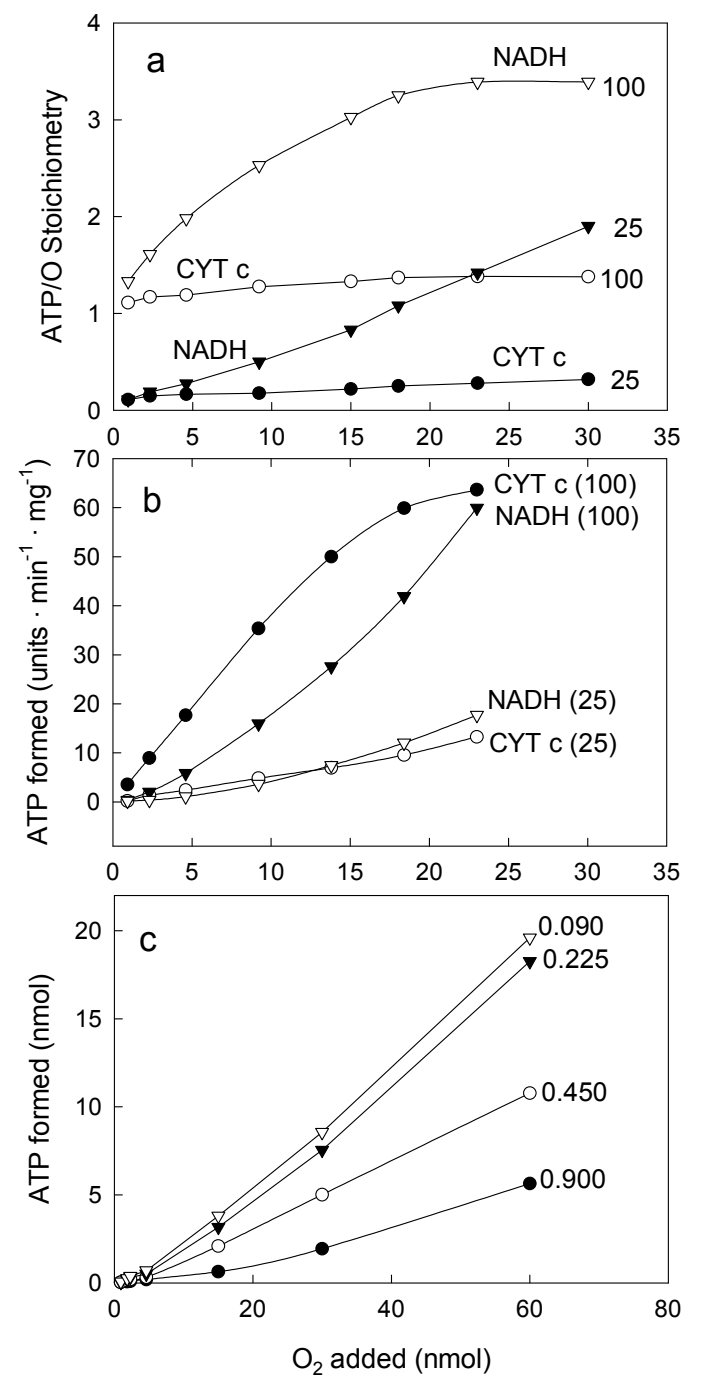

Figure 4. The ATP/O stoichiometry depends on all, the $\triangle E_{h}$ and the relative concentrations of $A D P, O_{2}$ and protein. The reaction mixtures were as described under Experimental Procedures. In Fig. 4 ( $a$ and $b$ ), the reactions were initiated by adding from 0.92 to $30.0 \mathrm{nmols}$ of $\mathrm{O}$ to fully reduced suspensions of $0.009 \mathrm{mg}$ of SMP supplemented with either $5 \mathrm{mM}$ NADH or cytochrome $c$ in the presence of 100 and $25 \mu \mathrm{M}$ ADP. The ATP/O ratio in Fig. 4a was determined at the moment in which the net synthesis of ATP ceased and the fast initial phase of $\mathrm{O}_{2}$ consumption was abruptly interrupted (see Figs. 1 and 2). The arithmetical means $\pm \mathrm{SD}_{\mathrm{n}-1}$ of at least 5 independents determinations performed at $\mathrm{O}_{2}$ concentrations of 5,10 and $15 \mu \mathrm{M}$ had a statistical significance " $P$ " $<0.05$. Error bars were eliminated to improve the Fig. In Fig. 4b, the rates of ATP synthesis were determined during the first $500 \mathrm{~ms}$ of reaction by measuring the steepest portion of the traces. Each unit represents $1 \mu$ mole in the presence of cytochrome $\mathrm{c}$ and $3.57 \mu$ mole in the presence of NADH. In Fig. $4 \mathrm{c}$ the extent of ATP synthesis was determined in reactions initiated by adding from 0.46 to $60 \mathrm{nmols} O$ to anaerobic and fully reduced suspensions of $0.09,0.225,0.45$ and $0.9 \mathrm{mg}$ of SMP protein in the presence of $100 \mu \mathrm{M}$ ADP and 5.0 mM NADH.

\section{$V$. The free energy of electron flow is essential not only for the binding or release of substrates and products but also for the synthesis of ATP from ADP and Pi.}

It was impressibly asserted that the covalent structure of ATP can be readily formed in the presence or absence of substrates or of oxidation inhibitors [5-8]. Figure 5 show, however, that even in the presence of very low levels of ATP and high of $\mathrm{O}_{2}, \mathrm{Pi}$ and ADP (optimal conditions for a spontaneously synthesis of ATP during an equilibrium period) the actual synthesis does not occur if there is no net flow of electrons. Instead, the hydrolysis of a miniscule amount of ATP (a contaminant of the sample of ADP) takes precedence over the actual synthesis of ATP, a process that continuous until a seemingly endless period of equilibrium is attained in which the rates of synthesis and hydrolysis of ATP are exactly the same [16]. This period of equilibrium is only interrupted when succinate is added and the free energy of electron flow brings about the actual synthesis of ATP from the ADP and Pi already bound to the membrane. It is evident that, when the mitochondria are incubated "with Pi labeled with ${ }^{18} \mathrm{O}$ and ${ }^{32} \mathrm{P}$ and unlabeled ATP in the presence or absence of substrates or of oxidation inhibitors" [8], ${ }^{18} \mathrm{O}$ is incorporated into Pi during the period of equilibrium in which the synthesis and hydrolysis of ATP are equal. What is remarkable in Fig. 5 is that, even in the presence of very high levels of $\mathrm{O}_{2}(\sim 230$ $\mu \mathrm{M})$ and $\operatorname{ADP}(400 \mu \mathrm{M})$ the initial rate of ATP synthesis is only $12.37 \mathrm{nmols} \cdot \mathrm{min}^{-1} \cdot \mathrm{mg}^{-1}$, i.e., $\sim 10^{3}$ times lower than in Figs. 1 and 2. Obviously, under state-3 metabolic conditions the mitochondrial membrane is not under optimal conditions, most likely due to the impairing effect of reactive oxygen species (see above). Indeed, these results demonstrate that the 
free energy of electron flow is essential not only for the binding and release of substrates and products to and from the ATP synthase but most importantly for the synthesis of ATP from ADP and Pi.

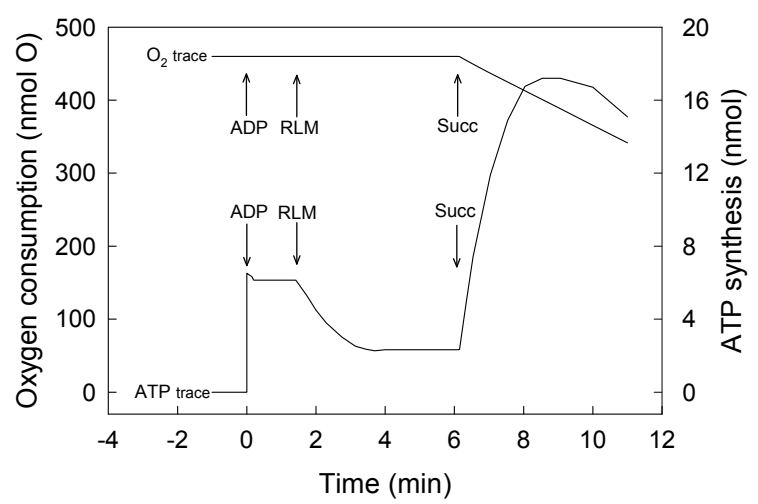

Figure 5. Demonstration that the free energy of electron flow is indispensable for the actual synthesis of ATP from ADP and Pi. The medium was that described under Experimental Procedures. The experiment was initiated by adding 400 nmols of ADP and 6.3 nmols of ATP (as contaminant of ADP) to an air-saturated medium free from RLM and succinate. After $1.5 \mathrm{~min}$ of incubation, $1.0 \mathrm{mg}$ of RLM protein was added to initiate the hydrolysis of the 6.3 nmols of ATP that proceed without the uptake $\mathrm{O}_{2}$ until a seemingly endless state of equilibrium was attained. This period of equilibrium was only interrupted when either succinate $(10 \mathrm{Mm})$ was added to initiate the simultaneous processes of $\mathrm{O}_{2}$ uptake and ATP synthesis or the concentration of $\mathrm{O}_{2}$ was near zero.

\section{The concentration of ADP required for half maximal response of ATP synthesis is an inverse function of both $\Delta E_{h}$ and $O_{2}$ concentration.}

For the first time evidence is here provided that, contrary to what is generally believed, the concentration ADP at which the rate of ATP synthesis is half its maximal value is not constant but varies subtly depending on both $\Delta \mathrm{E}_{\mathrm{h}}$ and $\mathrm{O}_{2}$ concentration. Unlike the hyperbolical hydrolysis of ATP that is entirely independent of $\Delta E_{h}$ and $\mathrm{O}_{2}$ [16], Fig. 6a show that for same concentration of ADP the initial rates of ATP synthesis increase directly depending on both $\Delta E_{h}$ and $\mathrm{O}_{2}$ concentration [35]. Figure $6 \mathrm{~b}$ demonstrates that the concentration of ADP required for half maximal rates of ATP synthesis $\left(E C_{50}\right)$ is an inverse function of $\Delta E_{h}$ and $\mathrm{O}_{2}$, decreasing from 76.0 to $36.7 \mu \mathrm{M}$ when both the concentration of $\mathrm{O}_{2}$ and the magnitude of $\Delta \mathrm{E}_{\mathrm{h}}$ increase. It is remarkable that the $E C_{50}$ for ADP is the same $(41.0 \mu \mathrm{M})$ whether in the presence of cytochrome c or NADH only when the concentration of $\mathrm{O}_{2}$ in the presence of cytochrome $\mathrm{c}$ is 5 fold higher than in the presence of NADH. The Hill coefficient, $n$, on the other hand, has a constant value of $\sim 3.0$ that is entirely independent of $\Delta E_{h}$ and $\mathrm{O}_{2}$ concentration. These results contrast assertions that the sigmoidal synthesis of ATP and the hyperbolical hydrolysis of ATP are mechanistically identical [7].
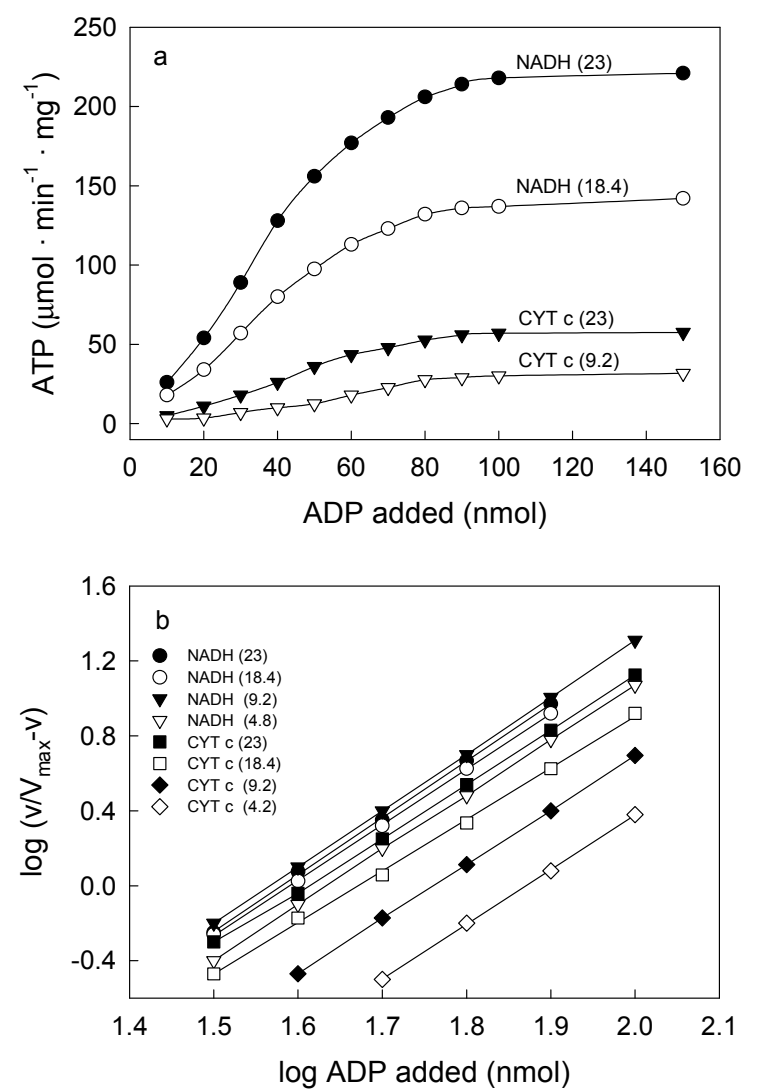

Figure 6. The concentration of ADP at which the rate of ATP synthesis is half its maximal value is regulated by both $\mathrm{O}_{2}$ and $\Delta E_{h}$. Reactions were initiated by adding from 4.6 to $23.0 \mathrm{nmols}$ $\mathrm{O}$ (figures in parenthesis) to anaerobic and fully reduced samples of $0.01 \mathrm{mg}$ of SMP in the presence of either $5.0 \mathrm{mM}$ NADH or $100 \mu \mathrm{M}$ cytochrome $\mathrm{c}$ and the indicated amount of ADP (x-axis). The same type of sigmoidal curve was obtained by comparing the amount of ADP initially present with either the initial rates of ATP synthesis (Fig. 6a) or the maximal amounts of ATP formed. Figure $6 \mathrm{~b}$ shows that the Hill coefficient, $n\left(\sim 3\right.$.) is a constant that is independent of $\Delta E_{h}$ or amount of $\mathrm{O}_{2}$ added. The concentration of ADP that produces half-maximal rates $\left(E C_{50}\right)$ or extents $\left(\mathrm{K}_{0.5}\right)$ of ATP synthesis can be calculated from equation 1 when the $\log \left(\mathrm{v} / \mathrm{V}_{\max }-\mathrm{v}\right)=0$.

\section{Conclusions}

1. The phosphorylation of ADP and the net synthesis of ATP cannot occur in the absence of a respiratory substrate and the net flow of electrons $(\Delta \Psi)$ toward oxygen.

2. The synthesis of ATP from ADP and Pi can efficiently take place in the absence of a proton gradient and the chemical component $(\Delta p H)$ of the protonmotive force, $\Delta p$.

3. The level of $\mathrm{O}_{2}$, not the level of ADP, is the most important factor in determining the rate of oxidative phosphorylation. 
4. The ATP/O stoichiometry is not constant but varies depending on all, the $\left(\Delta E_{h}\right)$, the redox state of the membrane and the relative levels of $\mathrm{ADP}, \mathrm{O}_{2}$ and protein.

5. The concentration of ADP at which the extent and rates of ATP synthesis is half maximal is not constant but decreases as the $\Delta E_{h}$ and the concentration of $\mathrm{O}_{2}$ increase.

6. The energy metabolism of the cell cannot be adequately evaluated by determining the mitochondrial $\mathrm{H}^{+} / \mathrm{O}$ ratio or the amount of $\mathrm{O}_{2}$ consumed under steady-3 metabolic conditions.

\section{Acknowledgments}

This research was supported in part by FONDECYT grant $\mathrm{N}^{\circ}$ 1061086. The authors express also their sincere gratitude to Dr. Peter L. Pedersen, Department of Biological Chemistry Johns Hopkins University, for providing reagents and sub-mitochondrial particles and to Dr. Sally $\mathrm{H}$. Cavanaugh, Department of research York Hospital, PA, for allowing the use of equipment.

\section{Conflict of interest}

The authors have declared that no conflict of interest exists.

\section{References}

1. Mitchell P. Coupling of phosphorylation to electron and hydrogen transfer by a chemiosmotic type of mechanism. Nature. 1961; 191: 144-8.

2. Brand MD. The stoichiometry of proton pumping and ATP synthesis in mitochondria. The Biochemist. 1994; 16: 20-4.

3. Chance B and Williams GR. The Respiratory Chain and Oxidative Phosphorylation. Adv Enzymol Relat Subj Biochem. 1956; 17: 65-134.

4. Berg JM, Tymoczko JL and Stryer L. The rate of oxidative phosphorylation is determined by the need for ATP. In: Biochemistry, $5^{\text {th }}$ Edition. New York, USA: Freeman WH \& Co. 2002:552.

5. Boyer PD, Cross RL and Momsen W. A new concept for energy coupling in oxidative phosphorylation based on a molecular explanation of the oxygen exchange reactions. Proc Natl Acad Sci USA. 1973; 70: 2837-39.

6. Kayalar C, Rosing J and Boyer PD. An alternating site sequence for oxidative phosphorylation suggested by measurement of substrate binding patterns and exchange reaction inhibitions. J Biol Chem. 1977; 252: 2486-91.

7. Milgrom YM and Cross RL. Nucleotide-depleted beef heart F1-ATPase exhibits strong positive catalytic cooperativity. J Biol Chem. 1997; 272: 32211-14.

8. Boyer PD. A research journey with ATP synthase. J Biol Chem. 2002; 277: 39045-61.

9. Brand MD and Murphy MP. Control of electron flux through the respiratory chain in mitochondria and cells. Biol Rev Camb Philos Soc. 1987; 62: 141-93.

10. Pedersen PL, Greenwalt JW, Reynafarje BD, Hullihen J, Decker GL, Sopper JW and Bustamante E. Preparation and Characterization of Mitochondria and Submitochondrial Particles of Rat Liver and Liver-Derived Tissues. Methods Cell Biol. 1978; 20: 411-81.

11. Napolitano MJ and Shain DH. Quantitating adenylate nucleotides in diverse organisms. J Biochem Biophys Methods. 2005; 63: 69-77.

12. Davies PW and Grenell RG. Metabolism and function in the cerebral cortex under local perfusion, with the aid of an oxygen cathode for surface measurement of cortical oxygen consumption. J Neurophysiol. 1962; 25: 651-83.

13. Reynafarje BD and Davies WP. The polyphasic nature of the respiratory process at the mitochondrial level. Am J Physiol 1990; 258: C504-C511.

14. Timmins GS, Robb FJ, Wilmot CM, Jackson SK and Swartz HM. Firefly flashing is controlled by gating oxygen to light-emitting cells. J Exp Biol. 2001; 204: 2795-801.

15. Bourgois JJ, Slues FE, Baguet $F$ and Mallefet J. Kinetics of light emission and oxygen consumption by bioluminescent bacteria. J Bioenerg Biomembr. 2001; 33: 353-63.

16. Reynafarje BD and Pedersen PL. ATP synthase. Conditions under which all catalytic sites of the $\mathrm{F}_{1}$ moiety are kinetically equivalent in hydrolyzing ATP. J Biol Chem. 1996; 271: 32546-50.

17. Lemasters JJ and Hackenbrock CR. Continuous measurement of adenosine triphosphate with firefly luciferase luminescence. In: Parker L and Fleischer S, eds. Biomembranes. New York: Academic Press; 1997: 703-16.

18. Mitchell P and Moyle J. Respiration-driven proton translocation in rat liver mitochondria. Biochem J. 1967; 105: 1147-1162.

19. Brand MD, Reynafarje BD and Lehninger AL. Stoichiometric relationship between energy-dependent proton ejection and electron transport in mitochondria. Proc Natl Acad Sci USA. 1976; 73: 437-441.

20. Reynafarje BD. The polyphasic reduction of oxygen to water by purified cytochrome c oxidase. Biochem Biophy Res Commun. 1991; 176: 150-6.

21. Ganong FW. Gas Transport between the Lungs \& the Tissues. In: Appleton \& Lange, eds. Physiology, 6th ed. Norwalk, Connecticut. 1993: 604-5.

22. Wittenberg BA and Wittenberg JB. Oxygen pressure gradients in isolated cardiac myocytes. J Biol Chem. 1985; 260: 6548-54.

23. Chance B and Williams GR. Respiratory enzymes in oxidative phosphorylation. I. Kinetics of oxygen utilization. J Biol Chem. 1955; 217: 383-93.

24. Wilson DF, Owen CS and Holian A. Control of mitochondrial respiration: a quantitative evaluation of the roles of cytochrome c and oxygen. Arch Biochem Biophys. 1977; 182:749-62.

25. Erecinska $M$ and Wilson DF. Regulation of cellular energy metabolism. J Membr Biol. 1982; 70: 1-14.

26. Le SB, Hailer MK, Buhrow S, Wang Q, Fatten K, Pediaditakis $P$, Bible KC, Lewis LD, Sausville EA, Pang YP, Ames MM, Lemasters $\mathrm{JJ}$ and Holmuhamedov EL. Inhibition of mitochondrial respiration as a source of adaphostin-induced reactive oxygen species and cytotoxicity. J Biol Chem. 2007; 282: 8860-72.

27. Kannt A, Lancaster CR and Mitchell HJ. The role of electrostatic interactions for cytochrome c oxidase function. J Bioenerg Biomembr. 1998; 30: 81-7.

28. Hill BC and Greenwood C. The reaction of fully reduced cytochrome c oxidase with oxygen studied by flow-flash spectrophotometry at room temperature. Evidence for new pathways of electron transfer. Biochem J. 1984; 218: 913-21.

29. Hill BC. The reaction of the electrostatic cytochrome c-cytochrome oxidase complex with oxygen. J Biol Chem. 1991; 266: 2219-26.

30. Reynafarje BD and Ferreira J. Cytochrome c oxidase: the mechanistic significance of structural $\mathrm{H}+$ in energy transduction. J Bioenerg Biomembr. 2002; 34: 259-67.

31. Reynafarje BD. Work and Oxygen Debt at Altitude-Biochemical Aspects. In: Helfferich C, ed. FT Wainwright, Alaska: Artic Aeromedical Laboratory. 1986: 105-9.

32. Reynafarje BD and Marticorena E. Bioenergetics of the heart at 
high altitude: environmental hypoxia imposes profound transformations on the myocardial process of ATP synthesis. J Bioenerg Biomembr. 2002; 34: 407-12.

33. Reynafarje $\mathrm{BD}$ and Lehninger $\mathrm{AL}$. The $\mathrm{K}+/$ site and $\mathrm{H}+/$ site stoichiometry of mitochondrial electron transport. J Biol Chem. 1978; 253: 6331-4.

34. Perez JA and Ferguson SJ. Kinetics of oxidative phosphorylation in Paracoccus denitrificans. 2. Evidence for a kinetic and thermodynamic modulation of F0F1-ATPase by the activity of the respiratory chain. Biochemistry 1990; 29: 10518-26.

35. Chung Y, Molé PA, Sailasuta N, Tran TK, Hurd R and Jue T. Control respiration and bienergetics during muscle contraction. Am J Physiol. 2005; 288: C730-8. 